\title{
Perceived Risk of Chlamydial and Gonococcal Infection Among Sexually Experienced Young Adults In the United States
}

By Carol A. Ford, James Jaccard, Susan G. Millstein,

Philip E. Bardsley and William C. Miller

Carol A. Ford is associate professor, Departments of Pediatrics and Medicine, Philip E. Bardsley is research associate, Carolina Population Center, and William C. Miller is associate professor, Departments of Medicine and

Epidemiology, all at the University of North Carolina, Chapel Hill. James Jaccard is professor, Department of Psychology, State University of New York, Albany. Susan G. Millstein is professor, Department of Pediatrics, University of California, San Francisco.

CONTEXT: Understanding young adults' perceived risk of chlamydial and gonococcal infection can inform interventions to reduce the prevalence of these sexually transmitted diseases.

METHODS: Bivariate and multivariate analyses, using data from Wave 3 of the National Longitudinal Study of Adolescent Health (2001-2002), were conducted to examine relationships between perceived risk and selected characteristics in two groups: a nationally representative sample of sexually experienced 18-26-year-olds and a subsample of those who tested positive for chlamydia or gonorrhea. The relationship between current infection and perceived risk was also evaluated.

RESULTS: Only 14\% of all respondents and 33\% of infected participants reported some perceived risk of chlamydial or gonococcal infection. In the overall sample, the odds of perceiving risk were significantly elevated among blacks, Hispanics, unmarried respondents, inconsistent condom users and nonusers, respondents who reported multiple partners in the past year, those who had received a diagnosis of chlamydia or gonorrhea in the past year, and those reporting current symptoms (odds ratios, 1.5-3.3). Currently infected participants were significantly more likely than those who were not infected to perceive some risk of infection (2.4). Among infected respondents, the factors positively associated with perceived risk were being black or Hispanic, using condoms inconsistently or not using them, having exchanged money for sex, having been tested in the past year but with no diagnosis, having received a diagnosis, and reporting current symptoms (2.5-5.2).

CONCLUSIONS: Interventions to increase the accuracy of young adults' risk perceptions may influence sexual and health care-seeking behaviors in a way that will reduce rates of chlamydia and gonorrhea.

Perspectives on Sexual and Reproductive Health, 2004, 36(6):258-264

Chlamydia trachomatis causes the most common bacterial sexually transmitted disease (STD) in the United States. ${ }^{1}$ Five percent of U.S. women aged 18-26 and 4\% of men that age have chlamydia; prevalences are elevated among black women (14\%), black men (11\%) and Hispanic men (7\%) in this agegroup. ${ }^{2}$ In comparison, the overall prevalence of gonococcal infection is low (0.4\%), but is elevated among blacks (2\%). These results, from Wave 3 of the National Longitudinal Study of Adolescent Health (Add Health), are consistent with findings from a growing literature that has documented wide racial disparities in rates of HIV and other STDs. ${ }^{3}$

In women, Chlamydia trachomatis and Neisseria gonorrhoeae can cause acute and long-term morbidity, including pelvic inflammatory disease, infertility from scarring of the fallopian tubes, chronic pelvic pain and ectopic pregnancy. ${ }^{4}$ Chlamydial infection may also be linked to an increased risk of cervical cancer, ${ }^{5}$ and both chlamydial and gonococcal infections may increase transmission of HIV in men and women. ${ }^{6}$ The direct medical costs associated with chlamydial and gonococcal infections among 15-24-year-olds in the United States in 2000 were an estimated $\$ 325$ million. $^{7}$

Perceived risk of infection is an important aspect of efforts to reduce the prevalence of chlamydia and gonorrhea for two reasons. First, risk perceptions can influence sexual behav- ior. Several theoretically driven STD intervention programs seek to change sexual behavior in part by altering risk perceptions. ${ }^{8}$ Although empirical data on perceived risk and condom use have yielded mixed results, in a prospective study of 14-19-year-olds attending an STD clinic, Ellen and colleagues found that participants' perceived risk of being infected by a main partner was an independent predictor of condom use with that partner. ${ }^{9}$ Few studies have examined the association between sexual behavior and perceived risk of current infection, but the two may be related. For example, a person who thinks he or she could be infected may be more likely than someone who does not to abstain or use condoms because of concerns about transmitting infection.

Second, risk perceptions can influence health careseeking behavior. ${ }^{10}$ A person who thinks he or she could have an STD may be more likely to seek a doctor or nurse's evaluation than someone with no perceived risk. Health care could be highly effective in reducing the prevalence of chlamydia and gonorrhea, because both infections are easy to diagnose and curable with a single dose of oral antibiotics. ${ }^{11}$ Early detection and treatment prevents complications and stems the spread of infection through sexual networks.

Risk perceptions are complicated by the fact that many STDs do not produce physical symptoms. More than 95\% 
of Wave 3 Add Health respondents who tested positive for chlamydia reported no symptoms within the previous 24 hours; findings for gonorrhea were similar. ${ }^{12}$ Within this context, it is important to understand risk perceptions among asymptomatic populations in nonclinical settings. However, most of what we currently know about perceived risk of STDs comes from clinic-based or regional studies. ${ }^{13}$

Wave 3 of Add Health provides a unique opportunity to increase our understanding of perceived risk because it is based on a nationally representative sample and included biological tests for chlamydia and gonorrhea. In this study, we used bivariate and multivariate analyses to examine relationships between perceived risk and respondents' demographic characteristics, sexual risk behaviors and other STD-related factors. Analyses were conducted among two groups: sexually experienced Wave 3 respondents aged 18-26 and a subsample of respondents who tested positive for chlamydia or gonorrhea at the time of the survey. We also examine the association between Wave 3 test results and perceived risk of infection.

\section{METHODS \\ Study Design}

Add Health is a prospective cohort study designed to follow almost 20,000 participants from adolescence to adulthood. Add Health sampling methods have been described in detail elsewhere. ${ }^{14}$ In brief, the original sample (Wave 1) was selected from enrollment rosters for grades 7-12 early in the 1994-1995 school year. The rosters came from 80 high schools and 52 middle schools. Schools were selected to be representative of U.S. schools in regard to region, urbanization, type of school, proportion of white students and size of school. Black students from relatively affluent families and certain Hispanic groups were oversampled to increase the precision of estimates for these groups. Appropriate weighting and analytic techniques that account for the sampling design can be used to ensure that the Add Health cohort provides a nationally representative study sample.

Wave 3 was conducted between August 2001 and April 2002. All Wave 1 respondents who were living in one of the 50 states and could be contacted were asked to participate in an interview and to provide a urine specimen for STD testing. Our study is based on the 11,821 Wave 3 respondents who were sexually experienced and provided a response to our measure of perceived risk, and for whom weights were available. Sexual experience was established by a positive response to the question "Have you ever had vaginal intercourse? (Vaginal intercourse is when a man inserts his penis into a woman's vagina.)."

\section{Procedures}

Wave 3 interviewers traveled to each respondent's home or to another suitable location identified by the potential participant. After respondents gave written consent, 90-minute interviews were conducted in as private an area as possible. Interviewers entered responses directly into a computer, and participants used computer-assisted self-interview to answer potentially sensitive questions (e.g., questions about sexual behavior and perceived risk of STDs).

At the end of the interview, participants were asked to consent to STD testing. Those who provided written consent were instructed to collect $20 \mathrm{ml}$ of first-void urine in specimen containers. Urine samples were placed in coolers, maintained at approximately $4^{\circ} \mathrm{C}$ until being packaged with fresh ice, and shipped by overnight express to a laboratory. ${ }^{15}$ All samples were processed on the day of receipt for Chlamydia trachomatis and Neisseria gonorrhoeae. Trained laboratory technologists performed ligase chain reaction assays according to the manufacturer's instructions (except they also tested specimens exceeding the recommended volume of $20 \mathrm{ml}$ ).

Participants who provided a urine specimen received information about chlamydia, gonorrhea and other STDs, and were encouraged to call a toll-free number to obtain their test results. These respondents were also informed that they were not being tested for all STDs and should not view their participation in the Add Health study as a substitute for health care.

The University of North Carolina Institutional Review Board approved all study procedures.

\section{Measures}

- Outcome variable. During the interview, participants were told that they would be asked to provide a urine sample to be tested for gonorrhea and chlamydia, and that they would be given a toll-free number to call for test results. The next item measured perceived risk of infection: "What is the chance that right now you have either gonorrhea or chlamydia?" Response options were provided on a five-point semantic scale that ranged from very low risk to very high risk. We created a dichotomous measure of perceived risk by coding respondents who answered very low as having no perceived risk and all other respondents as having some perceived risk. - Independent variables. Gender, race and ethnicity were self-reported. Participants who said they were of Hispanic or Latino origin were coded as Hispanic. If participants reported more than one racial category, they were asked to identify the one category that best described their background. We used dates of interview and birth to categorize participants as aged 18-20, 21-23 or older than 23. We based our measure of family socioeconomic status on the item "Before you turned 18, did anyone in your household ever receive public assistance or welfare payments?" We coded respondents who answered yes as having low family socioeconomic status and those who said no as having middle to high family socioeconomic status.

We constructed a measure for current relationship status from a series of items about romantic and sexual relationships. We categorized respondents who reported that they were currently in a relationship according to whether they were married, cohabiting but not married, or dating (neither married nor cohabiting). Respondents who reported no current relationship were coded as such.

Add Health included a series of questions about respondents' sexual behaviors. The item we included to mea- 
TABLE 1. Percentage distribution of sexually experienced 18-26-year-olds and percentage perceiving themselves to be at risk of infection with chlamydia or gonorrhea, by selected characteristics; and odds ratios (and $95 \%$ confidence intervals) from analyses assessing relationships between respondents' characteristics and perception of risk-National Longitudinal Study of Adolescent Health, 2001-2002

\begin{tabular}{|c|c|c|c|c|}
\hline Characteristic & $\begin{array}{l}\% \\
(\mathrm{~N}=11,821) \dagger\end{array}$ & $\begin{array}{l}\% \text { with } \\
\text { perceived } \\
\text { risk‡ }\end{array}$ & $\begin{array}{l}\text { Unadjusted } \\
\text { odds ratio }\end{array}$ & $\begin{array}{l}\text { Adjusted } \\
\text { odds ratio }\end{array}$ \\
\hline Total & 100.0 & 13.5 & na & na \\
\hline \multicolumn{5}{|l|}{ Gender } \\
\hline Female (ref) & 49.7 & 12.4 & 1.0 & 1.0 \\
\hline Male & 50.3 & 14.7 & $1.22(1.07-1.40)^{* *}$ & $1.07(0.90-1.26)$ \\
\hline \multicolumn{5}{|l|}{ Race/ethnicity } \\
\hline White (ref) & 68.4 & 11.3 & 1.0 & 1.0 \\
\hline Black & 16.4 & 20.4 & $2.01(1.68-2.41)^{* * *}$ & $1.55(1.26-1.91)^{* * *}$ \\
\hline Hispanic & 11.1 & 17.7 & $1.69(1.30-2.20)^{* * *}$ & $1.73(1.27-2.34)^{* * *}$ \\
\hline Asian & 3.2 & 12.9 & $1.16(0.80-1.68)$ & $1.14(0.75-1.72)$ \\
\hline Native American & 0.8 & 12.4 & $1.11(0.58-2.14)$ & $0.85(0.31-2.32)$ \\
\hline \multicolumn{5}{|l|}{ Age } \\
\hline$>23$ (ref) & 39.1 & 11.9 & 1.0 & 1.0 \\
\hline $21-23$ & 34.0 & 14.1 & $1.21(1.01-1.45)^{*}$ & $1.07(0.87-1.30)$ \\
\hline $18-20$ & 27.0 & 15.2 & $1.32(1.07-1.64)^{* *}$ & $1.07(0.86-1.33)$ \\
\hline \multicolumn{5}{|c|}{ Family socioeconomic status } \\
\hline Middle/high (ref) & 83.8 & 13.2 & 1.0 & 1.0 \\
\hline Low & 16.2 & 15.5 & $1.20(1.01-1.43)^{*}$ & $1.11(0.91-1.36)$ \\
\hline
\end{tabular}

Current relationship status Married (ref) 19.2

Cohabiting $\quad 19.3$

Dating $\quad 32.1$

None

32.1
29.3

5.2

11.4

15.7

18.2

Condom use in past 12 mos.

$\begin{array}{ll}\text { All (ref) } & 21.8 \\ \text { Some } & 48.2\end{array}$

$\begin{array}{ll}\text { Some } & 48.2 \\ \text { None } & 30.0\end{array}$

\section{4 \\ 17.5}

10.1

Ever exchanged money for sex

No (ref) $\quad 95.1$
Yes

Yes

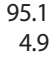

12.9

26.7

1.0

1.0

$2.34(1.68-3.27)^{* * *} \quad 1.97(1.40-2.78)^{* * *}$

$3.40(2.50-4.62)^{* * *} \quad 2.55(1.84-3.53)^{* * * *}$

$4.04(3.02-5.42)^{* * *} \quad 3.26(2.35-4.52)^{* * *}$

No. of partners in past 12 mos.

0 (ref) $\quad 8.2$

$1 \quad 58.7$

2

$\geq 3 \quad 17.3$

$\begin{array}{rll}9.0 & 1.0 & \\ 8.8 & 0.98(0.71-1.34) & 1.0 \\ 19.6 & 2.46(1.78-3.40)^{* * *} & 1.87(1.51-2.31)^{* * *} \\ 26.2 & 3.59(2.63-4.90)^{* * *} & 2.57(2.11-3.12)^{* * *}\end{array}$

Testing history for chlamydia or gonorrhea in past 12 mos.

Tested, no diagnosis

$80.0 \quad 12.3 \quad 1.0$

$1.0 \quad 1.0$

$1.65(1.37-1.98)^{* * *} \quad 1.69(1.40-2.05)^{* * *}$ $0.88(0.71-1.08) \quad 1.46(1.14-1.86)^{* *}$

Received diagnosis

Current symptoms

No (ref)

$16.3-14.8$

$3.7 \quad 36.4$

$1.24(1.02-1.52)^{*}$ $4.09(3.18-5.27)^{* * *}$

$1.00(0.77-1.30)$

94.1

5.9

12.7

25.4

1.0

${ }^{*} \mathrm{p} \leq .05$. ${ }^{* *} \mathrm{p} \leq .01$. ${ }^{* * *} \leq .001$. + Weighted percentages reflect the representative proportion in the U.S. population. ¥Percentages are based on weighted data and take into account Add Health's stratified sampling design to provide nationally representative results. Notes: ref=reference group. na=not applicable.

sure condom use was preceded by a question that asked participants how many times they had had vaginal intercourse in the past 12 months. Respondents who had had sex at least once during that time were asked "On how many of those occasions did (you/your partner) use a condom?" We coded participants according to whether they reported condom use on none, some or all occasions. We measured number of partners in the past year with the item "With how many different partners have you had vaginal intercourse in the past 12 months?" We categorized respondents as having had zero, one, two, or three or more partners. Because bivariate analyses revealed no significant differences in perceived risk between respondents who reported no partners in the past year and those who reported one partner, we combined these categories in the multivariate analyses. Exchange of money for sex was measured by two items: "Have you ever paid someone to have sex with you?" and "Have you ever had sex with someone who paid you to do so?" We coded respondents as having exchanged money for sex if they responded affirmatively to either question.

We based our measure of previous testing for chlamydia or gonorrhea on two items: "In the past 12 months, have you been told by a doctor or nurse that you have the following sexually transmitted diseases?" and "We'd like to know which, if any, of the following sexually transmitted diseases you have been tested for in the past 12 months." Chlamydia and gonorrhea were among the response options for both items. We placed participants in one of three categories: not tested for chlamydia or gonorrhea, tested but with no diagnosis, and having received a diagnosis.

The measure for current symptoms of potential infection was based on an item that presented a list of genitourinary symptoms and asked participants to identify those they had experienced in the last 24 hours. We categorized respondents as reporting current symptoms if they identified at least one of the following: "painful or very frequent urination (peeing)," "dripping or oozing from your (penis/vagina)," "bleeding after intercourse or between periods" and "itching in the vagina or genital area."

\section{Analysis}

Descriptive analyses used unweighted and weighted data. In the bivariate and multivariate analyses, we used data weighted to reflect a nationally representative sample and standard errors that were adjusted to account for the sample design. *

Our first set of analyses was based on the overall sample of 11,821 sexually experienced respondents. We used design-based Pearson F-statistics to examine bivariate relationships between perceived risk and respondents' demographic characteristics, sexual behaviors, STD testing history and reports of current symptoms. We then conducted survey multivariate logit analyses to evaluate the significance of each relationship when other factors were held constant.

Our second set of analyses focused on Wave 3 test results and perceived risk of infection. We conducted bivariate and multivariate analyses to determine the relationship between chlamydial and gonococcal test results and perceived risk of infection in the nationally representative sample. We then identified predictors of perceived risk among the 500 participants who tested positive for chlamydia or gonorrhea at the time of the survey, ${ }^{\dagger}$ using a similar analytic strategy to that described above.

*We adjusted standard errors using survey estimation commands in STATASE 8.

†Small sample sizes in subgroup analyses precluded inclusion of Native Americans. 


\section{RESULTS}

\section{Nationally Representative Sample}

Respondents in the overall sample were equally distributed by gender and roughly evenly distributed by age-group (Table 1). Sixty-eight percent were white, 16\% black, 11\% Hispanic, 3\% Asian and 1\% Native American. Sixteen percent came from families with low socioeconomic status, and only 19\% were married. Thirty percent of respondents said they had not used condoms during the last 12 months, and $5 \%$ had ever exchanged money for sex. The majority (59\%) said they had had only one partner in the past year, $16 \%$ had had two partners and 17\% had had three or more. Most respondents (80\%) had not been tested for chlamydia or gonorrhea in the 12 months preceding the survey, and $6 \%$ reported current symptoms.

Only 14\% of respondents thought that they had some risk of infection. At the bivariate level, respondents had significantly elevated odds of perceiving risk if they were male, were black or Hispanic, were in a young age-group, had low socioeconomic status, were unmarried, had used condoms inconsistently, had ever exchanged money for sex or had had multiple partners in the last 12 months (odds ratios, 1.2-4.0). Respondents who had been tested for chlamydia or gonorrhea in the past year but had not received a diagnosis, those who had received a diagnosis and those who reported current symptoms were also more likely than others to perceive some risk of infection (1.2-4.1).

In multivariate analyses, relationships persisted between perceived risk and race and ethnicity, relationship status, condom use, number of partners, previous diagnosis and reporting current symptoms. Blacks and Hispanics were significantly more likely than whites to think they were at risk of infection (odds ratios, 1.6 and 1.7, respectively). Compared with married respondents, those who were not married had significantly elevated odds of reporting some perceived risk (2.0-3.3). Respondents who reported inconsistent condom use, nonuse and multiple partners in the past 12 months were also more likely than comparison groups to perceive some risk of infection (1.5-2.6). Diagnosis of chlamydia or gonorrhea in the past year was significantly associated with perceived risk (2.4), as was reporting current symptoms (1.9).

We tested whether the strongest relationship observedthat between perceived risk and relationship status-was moderated by gender; it was not. We also analyzed associations between respondents' Wave 3 test results and their perceived risk of infection (not shown). The majority of respondents (67\%) who tested positive for chlamydia or gonorrhea did not perceive themselves to be at risk of infection. Nonetheless, in bivariate analyses, those with a positive test result were significantly more likely to report some perceived risk than were those with negative results (33\% vs. $13 \% ; \mathrm{F}=62.4$, p $\leq .001$ ). The relationship remained significant after we adjusted for all explanatory variables included in Table 1 (odds ratio, 2.4), and it was not moderated by gender or race and ethnicity.
TABLE 2. Percentage distribution of sexually experienced 18-26-year-olds who tested positive for chlamydia or gonorrhea, and percentage perceiving themselves to be at risk of infection, by selected characteristics; and odds ratios (and 95\% confidence intervals) from analyses assessing relationships between respondents' characteristics and perception of risk

\begin{tabular}{|c|c|c|c|c|}
\hline Characteristic & $\begin{array}{l}\% \\
(\mathrm{~N}=500) \dagger\end{array}$ & $\begin{array}{l}\% \text { with } \\
\text { perceived } \\
\text { risk‡ }\end{array}$ & $\begin{array}{l}\text { Unadjusted } \\
\text { odds ratio }\end{array}$ & $\begin{array}{l}\text { Adjusted } \\
\text { odds ratio }\end{array}$ \\
\hline Total & 100.0 & 33.1 & na & na \\
\hline \multicolumn{5}{|l|}{ Gender } \\
\hline Female (ref) & 57.7 & 29.3 & 1.0 & 1.0 \\
\hline Male & 42.3 & 38.2 & $1.49(0.83-2.68)$ & $1.75(0.86-3.57)$ \\
\hline \multicolumn{5}{|l|}{ Race/ethnicity } \\
\hline White (ref) & 33.7 & 17.6 & 1.0 & 1.0 \\
\hline Black & 50.0 & 41.4 & $3.30(1.49-7.32)^{* *}$ & $3.50(1.62-7.54)^{* *}$ \\
\hline Hispanic & 14.7 & 42.0 & $3.37(1.29-8.80)^{* *}$ & $3.93(1.39-11.12)^{* *}$ \\
\hline Asian & 1.6 & 20.2 & $1.18(0.16-8.68)$ & $2.06(0.22-19.10)$ \\
\hline \multicolumn{5}{|l|}{ Age } \\
\hline$>23$ (ref) & 32.3 & 31.2 & 1.0 & 1.0 \\
\hline $21-23$ & 35.9 & 28.3 & $0.87(0.46-1.67)$ & $0.83(0.38-1.77)$ \\
\hline $18-20$ & 31.8 & 40.4 & $1.49(0.73-3.04)$ & $1.28(0.61-2.68)$ \\
\hline \multicolumn{5}{|c|}{ Family socioeconomic status } \\
\hline Middle/high (ref) & 76.8 & 30.4 & 1.0 & 1.0 \\
\hline Low & 23.2 & 41.2 & $1.60(0.93-2.77)$ & $1.35(0.68-2.67)$ \\
\hline \multicolumn{5}{|c|}{ Current relationship status } \\
\hline Married (ref) & 11.8 & 28.5 & 1.0 & 1.0 \\
\hline Cohabiting & 14.8 & 23.1 & $0.75(0.22-2.55)$ & $0.72(0.20-2.55)$ \\
\hline Dating & 35.5 & 32.6 & $1.21(0.47-3.12)$ & $1.02(0.36-2.85)$ \\
\hline None & 37.8 & 39.0 & $1.61(0.68-3.79)$ & $1.26(0.45-3.54)$ \\
\hline \multicolumn{5}{|c|}{ Condom use in past 12 mos. } \\
\hline All (ref) & 24.3 & 24.3 & 1.0 & 1.0 \\
\hline Some & 51.3 & 37.7 & $1.89(0.69-5.19)$ & $2.52(1.13-5.62)^{*}$ \\
\hline None & 24.4 & 32.5 & $1.50(0.70-3.22)$ & $2.97(1.14-7.74)^{*}$ \\
\hline \multicolumn{5}{|c|}{ Ever exchanged money for sex } \\
\hline No (ref) & 89.8 & 30.1 & 1.0 & 1.0 \\
\hline Yes & 10.2 & 61.0 & $3.64(1.59-8.30)^{* *}$ & $5.22(1.68-16.25)^{* *}$ \\
\hline \multicolumn{5}{|c|}{ No. of partners in past 12 mos. } \\
\hline 07 & 2.6 & 18.5 & & \\
\hline 1$]$ ref & 48.6 & 25.1 & 1.0 & 1.0 \\
\hline 2 & 23.2 & 46.0 & $2.59(1.25-5.36)^{* *}$ & $1.92(0.87-4.22)$ \\
\hline$\geq 3$ & 25.5 & 34.8 & $1.63(0.87-3.04)$ & $1.43(0.71-2.88)$ \\
\hline \multicolumn{5}{|c|}{ Testing history for chlamydia or gonorrhea in past 12 mos. } \\
\hline Not tested (ref) & 70.9 & 26.9 & 1.0 & 1.0 \\
\hline Tested, no diagnosis & 18.8 & 39.2 & $1.75(0.88-3.46)$ & $2.78(1.00-7.70)^{*}$ \\
\hline Received diagnosis & 10.3 & 65.3 & $5.11(2.15-12.17)^{* * *}$ & $4.04(1.65-9.89)^{* *}$ \\
\hline \multicolumn{5}{|l|}{ Current symptoms } \\
\hline No (ref) & 91.5 & 30.5 & 1.0 & 1.0 \\
\hline Yes & 8.5 & 63.8 & $4.01(1.84-8.72)^{* * *}$ & $3.51(1.39-8.86)^{* *}$ \\
\hline
\end{tabular}

${ }^{*} \mathrm{p} \leq .05 .{ }^{* *} \mathrm{p} \leq .01 .{ }^{* * *} \leq .001$. + Weighted percentages reflect the representative proportion in the U.S. population. ¥Percentages are based on weighted data and take into account Add Health's stratified sampling design to provide nationally representative results. Notes: ref=reference group. na=not applicable.

\section{Infected Young Adults}

Among respondents who tested positive for chlamydia or gonorrhea, $58 \%$ percent were female; $34 \%$ were white, $50 \%$ black, 15\% Hispanic and 2\% Asian (Table 2). Respondents were evenly distributed by age-group. Twenty-three percent were from families with low socioeconomic status; $12 \%$ were married, $15 \%$ cohabiting, 36\% dating and 38\% not in a relationship. Twenty-four percent of infected respondents had not used condoms in the past 12 months, and $10 \%$ had ever exchanged money for sex. Almost half had 
had only one partner in the past year, and the remaining half were fairly evenly split between those reporting two partners and those reporting three or more. Seventy-one percent of currently infected participants had not been tested for chlamydia or gonorrhea in the past year, 19\% reported testing but no diagnosis and 10\% reported a positive diagnosis. Only 9\% reported current symptoms.

As noted above, one-third of participants who tested positive for chlamydia or gonorrhea perceived some risk of infection. Bivariate analyses revealed that respondents who were black or Hispanic, had exchanged money for sex, had had two partners in the past 12 months, had received a diagnosis of chlamydia or gonorrhea in the past year or reported current symptoms were significantly more likely than others to report some perceived risk (odds ratios 2.6-5.1).

All relationships remained significant in the multivariate analysis except the link between perceived risk and having had two partners. Moreover, associations between perceived risk and inconsistent condom use and previous testing became significant after we adjusted for other factors. Blacks and Hispanics were significantly more likely than whites to think they were at risk of infection (odds ratios, 3.5 and 3.9, respectively). Compared with respondents who had always used condoms in the last 12 months, inconsistent users and nonusers had elevated odds of perceiving some risk of infection (2.5 and 3.0). Having exchanged money for sex was significantly associated with perceived risk (5.2). Infected respondents who had been tested for chlamydia or gonorrhea in the past year but had not received a diagnosis were significantly more likely to perceive risk than were those who had not been tested (2.8); the same was true among respondents who had received a diagnosis in the past year (4.0). Participants who reported current symptoms were significantly more likely to perceive some risk of infection than those who were asymptomatic (3.5).

\section{DISCUSSION}

The vast majority of young adults in the United States think that their risk of chlamydial or gonococcal infection is very low-even if they engage in high-risk behaviors. Furthermore, the majority of young adults currently infected with chlamydia or gonorrhea do not think they are at risk. Atrisk and infected young adults who do not perceive themselves to be at risk may not modify their sexual behaviors or seek testing and treatment. This represents a tremendous public health challenge, especially given the unacceptably high rates of chlamydial infection among young people in this country.

From a health care perspective, our findings support current recommendations for routine annual chlamydial screening among all sexually experienced young women, ${ }^{16}$ and raise questions about why such screening is not recommended for men. Routine chlamydial testing of young adults is a control strategy that does not rely on perceived risk of infection. It is a mechanism by which young women and men can learn about infection and obtain treatment, even if they do not have physical symptoms or think they are at risk of infection. Diagnosis can trigger communication between sexual partners, treatment of partners and reduced prevalence of infection within sexual networks.

Our results also suggest that the current recommendations have not translated into widespread testing: Eighty percent of all respondents and $71 \%$ of infected respondents had not been tested for chlamydia or gonorrhea in the past year. A study of sexually experienced young adult women, who should all receive annual chlamydia testing under the current recommendations, yielded similar results. ${ }^{17}$ Future research will need to identify effective strategies to test and treat the large number of young adults in the United States who unknowingly have chlamydia or gonorrhea.

We are encouraged by our finding that currently infected young adults have higher odds of perceiving that they are at risk of infection than those who are not infected. Subgroups of blacks and Hispanics-both with disproportionately high rates of STDs-are more likely than whites to perceive some risk of infection. Furthermore, traditional behavioral indicators of increased STD risk (e.g., inconsistent condom use) are associated with increased odds of perceiving risk. These results provide evidence that, compared with their peers, young adults who are at increased risk of infection accurately perceive higher levels of risk.

Current relationship status and having had multiple partners in the past year were independently associated with perceived risk among respondents in the overall sample, but not among those who were currently infected. This difference may result from complex interactions between relationship status, number of partners, frequency of condom use and current infection status. Moreover, these interactions may vary by gender, race and other demographic and cultural factors. Although exploring these interactions is beyond the scope of our study, future research should do so.

Two of our findings suggest areas for further investigation that may be particularly relevant to service provision. First, previous diagnosis with chlamydia or gonorrhea was strongly associated with perceived risk, both in the overall sample and among currently infected respondents. Future studies should more closely examine the mechanisms by which diagnosis influences subsequent risk perceptions and the implications for sexual behavior, risk of repeat infection and ways to monitor for recurrent infection. Second, although only a small proportion of our sample reported current symptoms, such reports were positively associated with perceiving some risk of infection. This may be particularly helpful among males. The prevalence of chlamydial infection is $40 \%$ among young men in the general population who are experiencing symptoms of urethral discharge. ${ }^{18}$ Future research is needed to understand how to translate increased perceived risk of infection into increased testing, especially among symptomatic young men.

It is important to contextualize our study in the current literature on perceived risk. Brewer and colleagues have 
presented a useful model of risk perception and risk behavior. This model was designed to provide a general framework for studying the complex relationships between risk perceptions and behavior, and to show that ignoring these complex relationships has produced a body of inconsistent literature. ${ }^{19}$ Our finding that current infection is linked to perceived risk (i.e., that higher actual risk is associated with higher perceived risk) supports Brewer and colleagues' "accuracy hypothesis" with nationally representative data and biological test results. Because our study was crosssectional, we could not examine whether perceived risk influences sexual and health care-seeking behaviors (Brewer and colleagues' "behavioral motivation hypothesis") or whether changes in behavior influence risk perceptions (their "risk reappraisal hypothesis").

In a prior study of Wave 3 data, we examined the relationship between respondents' perceived risk of chlamydial or gonococcal infection and their willingness to consent to STD testing at the end of the interview. ${ }^{20}$ The relationship was not statistically significant, but this finding is difficult to interpret because Add Health participants received anonymous testing and financial incentives (neither of which would be available outside a research setting), and $92 \%$ provided a urine specimen. The relationship between respondents' perceived risk of infection and whether they called to obtain test results may more accurately reflect the effects of perceived risk on health care-seeking behaviors. This is the focus of our ongoing analyses.

Our study has several limitations. Because the original Add Health sample was drawn from school enrollment rosters, people who had dropped out of school before Wave 1 were not included. These individuals may be more likely than those who had stayed in school to engage in high-risk activities and may have different risk perceptions. However, Add Health has followed all Wave 1 participants, even those who dropped out of school, and previous research suggests that the potential bias because of missing school dropouts is small. ${ }^{21}$

Our study is also limited because the sample was restricted to respondents who reported a history of heterosexual vaginal intercourse; this excludes respondents who had participated in a range of other sexual behaviors associated with STD risk. In addition, our analyses that focused on currently infected respondents may be biased if this sample is significantly different from the group of Add Health respondents who were infected but refused to provide a urine specimen. We are in part reassured by previous findings that sexually experienced Wave 3 respondents who did not provide a urine specimen were similar to those who consented to testing with respect to gender, age, race and sexual behavior. ${ }^{22}$

Other potential limitations are related to measures. Wave 3 interviews included a single item to evaluate perceived risk of chlamydia or gonorrhea; this may have decreased the validity and internal consistency of our measure compared with measures based on multiple items. Many variables relied on self-reported data, which may not be fully accurate, although the use of computer-assisted selfinterview reduced the risk of underreporting for potentially sensitive questions. ${ }^{23}$ Reliance on recall may have weakened our measure of previous testing and diagnosis, although studies have demonstrated relatively high test-retest reliability among high school students for chlamydial testing in the past 12 months and pelvic examination at last checkup (kappas, 0.81 and 0.94 , respectively). ${ }^{24}$ In a study of 14-21-year-olds, the sensitivity and specificity of asking questions about chlamydial testing 4-6 months after a clinic visit were 100\% and 93\% respectively, which suggests that the validity of self-reported testing is excellent. ${ }^{25} \mathrm{Fi}$ nally, the tests used to detect chlamydia and gonorrhea perform well, but no test is perfect. Our assumption that participants who tested positive were infected may not be completely accurate, although previous estimates suggest that this inaccuracy is probably small. ${ }^{26}$

Despite these limitations, our results present an important portrait of perceived risk of chlamydial and gonococcal infection among young adults in the United States. The majority of young people who are at risk of infection do not realize or acknowledge that risk. Strategies to increase the accuracy of perceived risk are needed, especially among young people at the highest risk of infection. Successful approaches may include educational campaigns to raise awareness that many young people are infected with chlamydia but do not know it. It may be useful for such campaigns to include our finding that almost one-half of currently infected young adults had had only one partner in the last year, which shows that risk is not limited to people with multiple partners. Furthermore, parents may be in a position to help their young adult sons and daughters become aware of risks of STDs in a way that respects their children's developmental stage, and educational campaigns should consider including parents in the target audience. Any strategy to improve the accuracy of young adults' risk perceptions must be evaluated for efficacy and linked to studies that test how changes in perceived risk influence sexual and health care-seeking behaviors. In addition, there is an urgent need for strategies to reach the large number of currently infected young people who do not realize they are at risk of infection. As noted above, emphasizing routine screening for both women and men may be one way to achieve this. Routine screening in clinic settings could be substantially increased. Moreover, screening initiated outside clinic settings is now feasible through the use of urine samples and vaginal swab specimens, and this may provide opportunities to extend screening to high-risk populations that are not well connected to health care. ${ }^{27}$

\section{REFERENCES}

1. Centers for Disease Control and Prevention (CDC) Sexually Transmitted Disease Surveillance-2002, Atlanta: CDC, 2003.

2. Miller W et al., Prevalence of chlamydial and gonococcal infection among young adults in the United States, Journal of the American Medical Association, 2004, 291(18):2229-2236.

3. CDC, 2003, op. cit. (see reference 1); Hall H, Song R and McKenna M, Increases in HIV diagnoses-29 states, 1999-2002, Morbidity and Mortality Weekly Report, 2003, 52(47):1145-1148.

4. Cates W and Wasserheit J, Genital chlamydial infections: epidemi-
The majority of

young people

who are at risk

of infection do

not realize or

acknowledge

that risk. 
ology and reproductive sequelae, American Journal of Obstetrics and Gynecology, 1991, 164(6, part 2):1771-1781.

5. Wallin K et al., A population-based prospective study of Chlamydia trachomatis infection and cervical carcinoma, International Journal of Cancer, 2002, 101(4):371-374.

6. Fleming D and Wasserheit J, From epidemiological synergy to public health policy and practice: the contribution of other sexually transmitted diseases to sexual transmission of HIV infection, Sexually Transmitted Infections, 1999, 75(1):3-17.

7. Chesson HW et al., The estimated direct medical cost of sexually transmitted diseases among American youth, 2000, Perspectives on Sexual and Reproductive Health, 2004, 36(1):11-19.

8. Robin L et al., Behavioral interventions to reduce incidence of HIV, STD, and pregnancy among adolescents: a decade of review, Journal of Adolescent Health, 2004, 34(1):3-26.

9. Ellen JM et al., Adolescent condom use and perceptions of risk for sexually transmitted diseases-a prospective study, Sexually Transmitted Diseases, 2002, 29(12):756-762.

10. Brewer NT et al., Risk perceptions and their relationship to risk behaviors, Annals of Behavioral Medicine, 2004, 27(2):125-130.

11. CDC, Sexually transmitted diseases treatment guidelines-2002, Morbidity and Mortality Weekly Report, 2002, 51(RR-6):1-77.

12. Miller W et al., 2004, op. cit. (see reference 2).

13. Ellen JM et al., 2002, op. cit. (see reference 9); Kershaw TS et al., Misperceived risk among female adolescents: social and psychological factors associated with sexual risk accuracy, Health Psychology, 2003, 22(5):523-532; Ethier K et al., Adolescent women underestimate their susceptibility to sexually transmitted infections, Sexually Transmitted Infections, 2003, 79(5):408-411; and Ellen JM et al., Adolescents' perceived risk for STDs and HIV infection, Journal of Adolescent Health, 1996 18(18):177-181.

14. Resnick MD et al., Protecting adolescents from harm: findings from the National Longitudinal Study of Adolescent Health, Journal of the American Medical Association, 1997, 278(10):823-832.

15. Cohen MS et al., Biomarkers in Wave III of the Add Health study, University of North Carolina at Chapel Hill, 2003, <http://www. cpc.unc.edu/projects/addhealth/files/biomark.pdf>, accessed Nov. 1, 2004.

16. U.S. Preventive Task Force, Screening for chlamydial infection: recommendations and rationale, American Journal of Preventive Medicine, 2001, 20(3S):90-94; and CDC, 2002, op. cit. (see reference 11).

17. Iritani B et al., Assay results vs. self-reported chlamydial infections: does measurement discrepancy vary by level of risk behavior? paper presented at the National STD Prevention Conference, Philadelphia Mar. 8-11, 2004
18. Miller W et al., 2004, op. cit. (see reference 2).

19. Brewer NT et al., 2004, op. cit. (see reference 10).

20. Ford CA et al., Who refuses to provide a urine specimen for Chlamydia trachomatis and Neisseria gonorrhoeae testing? paper presented at the annual meeting of the Society for Adolescent Medicine, St. Louis, Mar. 25, 2004.

21. Udry JR and Chantala K, Missing school dropouts in surveys does not bias risk estimates, Social Science Research, 2003, 32(2):294-311.

22. Ford CA et al., 2004, op. cit. (see reference 20).

23. Turner $C$ et al., Adolescent sexual behavior, drug use, and violence: increased reporting with computer survey technology, Science, 1998, 280(5365):867-873

24. Santelli J et al., Reliability in adolescent reporting of clinician counseling, health care use, and health behaviors, Medical Care, 2002, 40(1):26-37.

25. Klein JD et al., Developing quality measures for adolescent care: validity of adolescents' self-reported receipt of preventive health services, Health Services Research, 1999, 34(1):391-404.

26. Cohen MS et al., 2003, op. cit. (see reference 15); and Miller W et al., 2004, op cit. (see reference 2).

27. Ford $\mathrm{C}$ et al., Testing for chlamydial and gonorrheal infections outside of clinic settings: a summary of the literature, Sexually Transmitted Diseases, 2004, 31(1):38-51.

\section{Acknowledgments}

Support was provided in part by The Robert Wood Johnson Foundation Generalist Physician Faculty Scholar Award program, the University of North Carolina STD Clinical Research Center, and the Clinical Associate Physician Program of the General Clinical Research Center, Division of Research Resources, National Institutes of Health. The authors thank Jonathan M. Ellen for having reviewed drafts of this article.

This research uses data from Add Health, a program project designed by J. Richard Udry, Peter S. Bearman, and Kathleen Mullan Harris, and funded by a grant P01-HD31921 from the National Institute of Child Health and Human Development, with cooperative funding from 17 other agencies. Special acknowledgment is due Ronald R. Rindfuss and Barbara Entwisle for assistance in the original design. Persons interested in obtaining data files from Add Health should contact Add Health, Carolina Population Center, 123 W. Franklin Street, Chapel Hill, NC 27516-2524 (<www.cpc. unc.edu/addhealth/contract.html>).

Author contact: caf@med.unc.edu 